\title{
Associação entre idade, classe social e hábito de fumar maternos com peso ao nascer*
}

\section{The association of maternal age, social class and smoking with birthweight}

\author{
Antonio A.M. da Silva", Uilho A. Gomes , Heloisa Bettiol Creusa M.R. Dal Bo Gerson \\ Mucillo Marco A. Barbieri
}

\begin{abstract}
SILVA, A.A.M. da et al. Associação entre idade, classe social e hábito de fumar maternos com peso ao nascer. Rev. Saúde públ., S. Paulo, 26: 150-4, 1992. Foi realizado inquérito em Ribeirão Preto, Brasil, de junho de 1978 a maio de 1979, que constou de entrevistas com mảes de nascidos vivos hospitalares, de partos únicos, correspondendo a $98 \%$ dos nascimentos ocorridos durante o período no Município. O maior percentual de nascimentos com baixo peso ocorreu entre as mães jovens, fumantes e pertencentes is classes trabalhadoras. $O$ maior número de mães jovens foi encontrado também nessas classes. A prevalência do hábito de fumar foi maior nas mães jovens, porém houve pouca diferença na frequência de mulheres fumantes entre as classes sociais, variando de 25 a $30 \%$. Observou-se que o pencentual de baixo peso foi maior para as mães trabalhadoras não fumantes do que para as mães burguesas fumantes. O modelo logito foi ajustado aos dados para estudo da possível associação múltipla entre hábito de fumar, idade materna e classe social com o peso ao nascer. Os resultados indicaram que hábito de fumar materno, idade materna e classe social foram independentes no seu efeito sobre o peso ao nascer, não se observando interação. Esses achados sugerem que a maior prevalência de baixo peso entre mães não fumantes das classes trabalhadoras em relaçåo às mães fumantes da burguesia provavelmente refletiu a concentração de outros fatores de risco entre as mulheres das classes trabalhadoras, tais como: baixa escolaridade, cuidado pré-natal inadequado, alta paridade, diferenças no comportamento reprodutivo.
\end{abstract}

Descritores: Baixo peso ao nascer. Idade materna. Fumo, efeitos adversos. Classe Social

\section{Introdução}

$O$ peso ao nascer determina, em grande parte, a sobrevida da criança no primeiro ano de vida e, mais freqüentemente, no primeiro mês ${ }^{24}$. Além do mais, a prevalência de baixo peso (peso de nascimento menor que $2.500 \mathrm{~g}$ ) é maior nos países subdesenvolvidos, onde cerca de $2 / 3$

* Parte do projeto "Estudo epiemiológico-social de parâmetros da saúde matemo-infanti" de responsabilidade dos Professores Doutores Marco Antonio Barbieri e Uilho Antonio Gomes da Faculdade de Medicina de Ribeirão Preto - USP, realizado de $1^{2}$ de junho de 1978 a 31 de maio de 1979.

* Departamento de Medicina III da Universidade Federal do Maranhão - São Luís, MA - Brasil.

*** Departamento de Medicina Social da Faculdade de Me dicina de Ribeirão Preto da USP - Ribeirão Preto, SP Brasil.

**** Departamento de Puericultura e Pediatria da Faculdade de Medicina de Ribeirāo Preto da USP - Ribeirão Preto, SP - Brasil.

*****Departamento de Geologia, Física e Matemática da Faculdade de Filosofia, Ciências e Letras de Ribeirão Preto da USP - Ribeirão Preto, SP - Brasil.

Separatas/Reprints: H. Bettiol - Av. Bandeirantes, 3900 14049-900 - Ribeirāo Preto, SP - Brasil.

Publicação financiada pela FAPESP. Processo Saúde Coletiva 91/4994-0. dessas crianças são a termo e sofreram retarde de crescimento intrauterino 5 . O peso ao nascer tem sido utilizado como indicador de desenvolvimento social ${ }^{25}$.

Muitas variáveis têm sido associadas com o peso ao nascer ${ }^{2,6,9,12}$. A prevalência de baixo peso é relatada como sendo maior nos recémnascidos de mães jovens ${ }^{13,16,17,18}$. O hábito de fumar materno tem sido, também, responsabilizado pelo baixo peso da criança ${ }^{21}$, parecendo que o seu efeito é do tipo dose-resposta ${ }^{11,14}$.

Alguns trabalhos têm mostrado a distribuição heterogênea do baixo peso entre subgrupos populacionais do mesmo país. Monteiro ${ }^{15}$, em 1976, mostrou uma prevalência global de baixo peso de 9,7\% nas maternidades de São Paulo. Este indice era menor nas maternidades privadas, $8,9 \%$, do que nas públicas, 12,1\%. Em 1983, o percentual de baixo peso nos Estados Unidos foi de $6,8 \%$; no entanto, a percentagem para os negros, $12,6 \%$, foi o dobro da dos brancos, $5,7 \%^{20}$.

O presente trabalho faz parte de um estudo mais amplo ${ }^{1}$ e nele se pretende estudar as relações entre o peso ao nascer, a idade, o hábito de fumar e classe social maternos. 


\section{Material e Método}

Foram coletados dados de nascidos vivos hospitalares, residentes e não-residentes, correspondendo a $98 \%$ do total. Para detalhes sobre a metodologia consultar publicação anterior'

A conceituação de classe social foi baseada no modelo proposto por Singer ${ }^{22}$ e adaptado para uso epidemiológico por Barros ${ }^{3}$, empregando indicadores que situam os indivíduos dentro das relaçōes sociais de produção, sendo que este conceito estabelece um conjunto de grupos "sócio-ocupacionais" que, reagrupados, permitem a discriminação das "frações de classe" na população estudada: a burguesia, proprietária dos meios de produção; o proletariado, que vende sua força de trabalho no mercado em troca de um salário; e o subproletariado, constituído dos demais trabalhadores com inserção débil no mercado de trabalho. A idade materna em anos completos e a informação sobre o hábito de fumar foram obtidas na entrevista com a mãe. Foi considerada fumante a mãe que consumiu qualquer número de cigartos durante a gestação.

A análise conjunta das variáveis foi realizada através do modelo logito ${ }^{23}$ e o qui-quadrado $\left(x^{2}\right)$ foi usado como medida de grau de associação ${ }^{10}$ e para testar a adequação do modelo. $O$ nivel de significância adotado foi sempre 0,05 .

\section{Resultados}

As crianças estudadas estão distribuidas na Tabela 1 segundo seu peso ao nascer, idade, classe social e hábito de fumar maternos.
No que diz respeito à idade, a proporção de baixo peso foi maior nas mulheres com idade inferior a 20 anos $\left(x^{2}=14,9, p<0,001\right)$.

$\mathrm{Na}$ mesma Tabela pôde-se observar que, entre as mäes com menos de 20 anos, as proporçōes de baixo peso segundo a classe social foram: 10,6\% (42/399) para o subproletariado, $8,8 \%(53 / 599)$ para o proletariado e $6,9 \%(5 / 72)$ para a burguesia. Embora pareça haver uma tendência nessas proporções, as diferenças observadas não foram estatisticamente significantes.

A proporção de mães jovens foi maior nas classes menos favorecidas, representando $14,0 \%$ no proletariado (599/4.267) e $17,5 \%$ no subproletariado (395/2.251), enquanto na burguesia representaram 5,0\% (72/1.437). Essas diferenças foram estatisticamente significantes $\left(x^{2}=129,6\right.$, $\mathrm{p}<0,01$ ).

A análise feita para o hábito de fumar materno mostrou um percentual de baixo peso maior para as mães fumantes $(9,6 \% ; 211 / 2.192)$ do que para as mães não fumantes $(5,4 \%$; $313 / 5.763)\left(x^{2}=45,5, p<0,01\right)$. Observando-se a Tabela 1, o percentual de baixo peso ao nascer foi maior para as mães dos grupos proletariado - 5,9\% (187/3.154) - e subproletariado - 6,3\% (98/1.562) - que não fumavam do que para as mães da burguesia que fumavam - 4,3\% $(17 / 390)\left(x^{2}=55,4, p<0,01\right)$. De qualquer modo, so foi observada a qualidade de fumante ou não fumante, não se analisando outros fatores que podem interfetir na relação, tais como quantidade de cigarros fumados ou hábito de fumar passivo, entre outros.

As prevalências de mães fumantes nas clas-

Tabela 1. Distribuição dos recém-nascidos estudados segundo seu peso ao nascer e idade, classe social e hábito de fumar maternos, bem como dos respectivos valores esperados segundo o modelo logito ajustado.

\begin{tabular}{|c|c|c|c|c|c|c|}
\hline $\begin{array}{l}\text { Idade } \\
\text { Materna }\end{array}$ & $\begin{array}{l}\text { Hábito } \\
\text { de Fumar }\end{array}$ & $\begin{array}{c}\text { Peso ao } \\
\text { Nescer }\end{array}$ & Burguesia & $\begin{array}{c}\text { Classe Social } \\
\text { Proletariado }\end{array}$ & Sub-Proletariado & Total \\
\hline \multirow[t]{2}{*}{$<20$} & Fumantes & $\begin{array}{l}<2.500 \\
\geq 2.500\end{array}$ & $\begin{array}{c}1(1,8) \\
\frac{29}{30}(28,2)\end{array}$ & $\begin{array}{c}23(23,9) \\
\frac{169}{192}(168,1)\end{array}$ & $\begin{array}{c}25(24,3) \\
\frac{145}{170}(145,8)\end{array}$ & $\begin{array}{r}49 \\
343 \\
392\end{array}$ \\
\hline & $\begin{array}{c}\text { Não } \\
\text { Fumantes }\end{array}$ & $\begin{array}{l}<2.500 \\
\geq 2.500\end{array}$ & $\begin{array}{c}4(1,4) \\
\frac{38}{42}(40,6)\end{array}$ & $\begin{array}{c}30(29,7) \\
\frac{377}{407}(377,3)\end{array}$ & $\begin{array}{c}17(19,0) \\
\frac{208}{225}(206,0)\end{array}$ & $\begin{array}{r}51 \\
623 \\
674\end{array}$ \\
\hline \multirow[t]{2}{*}{220} & Fumantes & $\begin{array}{l}<2.500 \\
\geq 2.500\end{array}$ & $\begin{array}{c}16(16,2) \\
\frac{344}{360}(343,8)\end{array}$ & $\begin{array}{c}83(87,8) \\
\frac{838}{921}(833,2)\end{array}$ & $\begin{array}{c}63(57,1) \\
\frac{456}{519}(461,9)\end{array}$ & $\begin{array}{r}162 \\
1.638 \\
1.800\end{array}$ \\
\hline & $\begin{array}{c}\text { Não } \\
\text { Fumantes }\end{array}$ & $\begin{array}{l}<2.500 \\
\geq 2.500\end{array}$ & $\begin{array}{r}24(25,6) \\
981(979,4) \\
1.005\end{array}$ & $\begin{aligned} & 157(151,6) \\
& 2.590(2.595,4) \\
& 2.747 \\
&\end{aligned}$ & $\begin{aligned} & 81(85,7) \\
& \frac{1.256}{1.337}(1251,3) \\
&\end{aligned}$ & $\begin{array}{r}262 \\
4.827 \\
5.089 \\
\end{array}$ \\
\hline Total & & & 1.437 & 4.267 & 2.251 & 7.955 \\
\hline
\end{tabular}


ses sociais foram $27,1 \%(390 / 1.437)$ na burguesia, $26,1 \%(1.113 / 4.267)$ no proletariado e $30,6 \%(689 / 2.251)$ no subproletariado $\left(x^{2}=\right.$ $15,3, \mathrm{p}<0,01)$. As mães com menos de 20 anos fumaram mais $(36,8 \% ; 392 / 1.066)$ do que as mais velhas $(26,1 ; 1.800 / 6.889)\left(x^{2}=52,4, \mathrm{p}<\right.$ 0,01).

Em relação à classe social, o baixo peso ao nascer foi cerca de duas vezes mais freqüente no proletariado - 6,9\% (293/4.267) - e quase 3 vezes maior no subproletariado - $8,3 \%$ $(186 / 2.251)$ - do que na burguesia - 3,1\% (45/1.437) $\left(\mathrm{x}^{2}=38,7, \mathrm{p}<0,01\right)$.

A Tabela 1 ainda apresenta as freqüências observadas e esperadas para o modelo que melhor se ajustou aos dados. Construiu-se um modelo logito com quatro variáveis; peso ao nascer como variável dependente, classe social, idade e hábito de fumar maternos como variáveis independentes. $O$ modelo que apresentou $O$ melhor ajuste aos dados foi o seguinte: $\log f_{i j k}=P+P$ $\mathrm{x} I+\mathrm{P} \times \mathrm{CS}+\mathrm{P} \times \mathrm{F}$, onde $\mathrm{P}$ é peso ao nascer, $\mathrm{CS}$ é classe social, $\mathrm{F}$ indica hábito de fumar materno e I representa idade materna. Não foi usado 0 modelo saturado porque as estatísticas $\times 2$ fornecidas pelo programa indicaram que o modelo, sem as interaçōes de maior grau, se ajustou adequadamente aos dados.

As estimativas dos parâmetros por ponto e intervalos de confiança, bem como os respectivos riscos relativos, encontram-se na Tabela 2.

Tabela 2. Estimativas dos parâmetros por ponto e intervalos de confiança, e riscos relativos para o modelo logito ajustado.

\begin{tabular}{cccc}
\hline Parâmetro & Estimativa & $\begin{array}{c}\text { Intervalo de } \\
\text { confianca a 95\% }\end{array}$ & $\begin{array}{c}\text { Risco } \\
\text { Relativo }\end{array}$ \\
\hline$P(1)$ & $\cdot 1,3058$ & $(-1,3769 ;-1,2347)$ & \\
$P(1) \times I(1)$ & 0,0744 & $(0,0166 ; 0,1322)$ & 1,6 \\
$P(1) \times F(1)$ & 0,1475 & $(0,1016 ; 0,1934)$ & 1,9 \\
$P(1) \times C S(1)$ & 0,1871 & $(0,1134 ; 0,2608)$ & 1,4 \\
$P(1) \times C S(2)$ & 0,1077 & $(0,0393 ; 0,1761)$ & 1,1 \\
\hline
\end{tabular}

$P(1)$ : baixo peso

I(1): idade materna $<20$ anos

$F(1)$ : mãe fumante

CS(1):subproletariado

CS(2): proletariado

Esse modelo ajustado indica que o peso ao nascer foi função de idade, classe social e hábito de fumar maternos, e estas variáveis não apresentaram interação entre si, fossem elas tomadas duas a duas ou três a três, mostrando que para qualquer classe social o hábito de fumar aumentou a probabilidade do nascimento de uma criança de abaixo peso; do mesmo modo, fosse a mãe fumante ou não, e para qualquer idade da mesma, a probabilidade de nascer uma criança de baixo peso aumentou com a piora da classe social.

O mesmo se aplica para a idade materna, ou seja, a mãe jovem teria probabilidade maior de ter um filho de baixo peso se ela fosse fumante, independentemente da classe social, e essa probabilidade também seria maior se ela pertencesse ao subproletariado, quer fosse fumante ou não.

\section{Discussão e Conclusões}

Observou-se uma associação entre hábito de fumar materno e peso ao nascer quando se tomou em consideração a população total. Becerra \& Smith ${ }^{4}$ sugerem que o efeito do hábito de fumar na ocorrência de baixo peso ao nascer pode ser modificado pela idade materna e pelas condiçōes sociais. No presente estudo, hábito de fumar, classe social e idade materna tomados simultaneamente, foram independentes entre si quanto ao seu efeito no peso ao nascer, podendo-se concluir que a situação social não influenciou no sentido de uma ação mais deletéria desse hábito nas classes trabalhadoras do que nas classes burguesas em relação ao peso ao nascer, nem nas mães mais jovens. Independentemente de sua classe social e idade, se uma mulher fuma, o recém-nascido deve ser afetado em seu peso.

Os resultados do presente estudo foram semelhantes aos relatados por Rush \& Cassano ${ }^{21}$, que, nas diferentes classes sociais, encontraram influência semelhante do hábito de fumar sobre o peso ao nascer. Observaram, porém, que a mortalidade perinatal em fumantes estava aumentada na classe de trabalhadores manuais.

Como explicar que mães não fumantes das classes trabalhadoras tiveram maior proporção de recém-nascidos de baixo peso em relação às mães fumantes das classes burguesas? A situação de serem mulheres das classes trabalhadoras faz com que tenham pior escolaridade, possuam um baixo nivel de rendimentos, consumam assistência médica em menor porporção e de pior qualidade, tenham maior número de filhos, experimentem uma situação conjugal desfavorável, entre outros. O conjunto desses fatores vai refletir-se em maior degeneração dos seus corpos, que determina um pior resultado biológico, no nascimento de um maior percentual de recémnascidos de baixo peso. Estes fatores, então, no seu conjunto, teriam efeito mais acentuado do que o próprio tabaco em si. 
Um fato que deve ser considerado é que no presente estudo a prevalência do hábito de fumar não variou significantemente entre as diferentes classes sociais (25 a $30 \%$, com média de $27,6 \%$ ). Bewley ${ }^{8}$, em um trabalho realizado na Inglaterra, mostrou que na classe dos operários não qualificados, as mulheres apresentavam maior prevalência do hábito, além de fumarem um maior número de cigarros. Isto confirma os resultados de outros autores, que mostram estar havendo decréscimo no hábito de fumar entre as mulheres das classes altas ${ }^{9,21}$. Ribeirão Preto parecia estar situação intermediária, em que algumas classes já estavam abandonando o hábito de fumar, talvez captando o discurso médico sobre os malefícios do tabaco, e outras ainda estavam incorporando o hábito como "status", como, por exemplo, no caso das mulheres entrando na força de trabalho. Este comportamento precisa de novas investigaçōes para ser melhor elucidado.

No caso da idade materna, observou-se maior proporção de baixo peso entre as mães com menos de 20 anos. Por sua vez, a proporção de mães jovens foi maior nas classes sociais menos favorecidas. Portanto, as mães desse grupo etário deveriam constituir-se grupo prioritário para a assistência pré-natal, em termos de saúde pública ${ }^{7,19}$. Lee e col. ${ }^{13}$ encontraram um padrão diferente na associação entre idade materna e peso ao nascer, estudando crianças a termo com 40 semanas de idade gestacional. Quando o risco para o nascimento de baixo peso de termo foi ajustado para algumas variáveis relacionadas com o comportamento reprodutivo, como raça, escolaridade, paridade, situação conjugal e cuidados pré-natais, o resultado mostrou risco menor para as mães adolescentes. Além do mais, este risco aumentou com o avanço da idade da mãe, sugerindo que este maior risco aparente de baixo peso ao nascer para a gravidez na adolescência seja reflexo de uma aglomeraçāo de outros fatores de risco nesta faixa etária, como os dados do presente estudo parecem indicar.

Pickering ${ }^{16}$ encontrou proporções maiores de baixo peso para a classe dos trabalhadores manuais. Estudando as primíparas, observou interação entre idade materna e classe social no risco de baixo peso, sendo que a idade de maior risco foi diferente em cada classe social, considerando 4 faixas etárias. No seu entender, estas diferenças se explicam pelo fato de que as mães diferentes classes sociais tendem a conceber em idades diferentes. Assim, apesar de variável biológica, a idade da mulher na procriação apresenta diferenças importantes no seu padrão de comportamento reprodutivo segundo o modo de vida das mulheres de diferentes classes sociais.

Para Pickering ${ }^{16}$, as variáveis sociais e biológicas estāo inter-relacionadas e a contribuição relativa de cada uma no risco de nascimento de baixo peso deve mudar com o tempo. Em relação à classe social, os resultados do presente trabalho mostram que, tanto no caso do hábito de fumar como da idade materna, as diferenças de classe devem ser levadas em consideração na explicação dos diferentes riscos e comportamentos biológicos dos indivíduos. A posição social condiciona série de aspectos da vida das pessoas que se refletem, por exemplo, nos padrōes de hábito de fumar e nas modalidades de comportamento reprodutivo que estão intimamente relacionados com o resultado final que a idade ou o hábito de fumar maternos provocam no peso ao nascer. Outros fatores também devem interferir no mesmo fenômeno, pois, independentemente destes dois estudados, há um efeito claro da classe social sobre o peso ao nascer.

SILVA, A.A.M. da et al. [The association of maternal age, social class and smoking with birthweight]. Rev. Saúde públ., S. Paulo, 26:150-4, 1992. An epidemiological survey was carried out in Ribeirão Preto, Brazil, from June 1978 to May

1979. Interviews were held with mother of singleton live borne children, delivered in hospitals, which accounted for $98 \%$ of all births in the area. The higher percentages of low birthweight children related to the offspring of smokers, young mother and women belonging to the working class. The majority of young women were found in the working class and the prevalence of smoking was higher in the group of women below 20 years of age. There was no statistical difference in the smoking habit as between different social classes. A larger number of low birthweight children were observed in nonsmoking women of the working class than among women smokers of the middle class. A log model was adjusted to the data in order to study the possible multiple association of smoking, maternal age and social class with birthweight. The results indicated that maternal smoking, maternal age and social class had independent effects on birthweight. The was no interaction between them. These findings suggest that the higher prevalence of low birthweight in nonsmoking mothers of the working class in relation to smoking mothers of the middle class probably reflects clustering of other risk factors-such as poor education inadequate prenatal care, high parity and differences in reproductive behavior in women of the working class.

Keywords: Infant, low birth weight. Maternal age. Smoking, adverse effects. Social class. 


\section{Referências Bibliográficas}

1. BARBIERI, M.A.; GOMES, U.A.; BARROS FILHO, A.A.; BETTIOL, H.; ALMEIDA, L.E.A; SILVA, A.A.M. Saúde perinatal em Ribeirão Preto, SP, Brasil: a questão do método. Cad Saúde públ., Rio de Janeiro, 5: 376-87, 1989.

2. BARROS, F.C.; VICTORA, C.G.; VAUGHAN, J.P.; ESTANISLAU, H.J. Bajo peso al nacer en el municipio de Pelotas, Brasil: factores de riesgo. Bol. Ofic. sanit. panamer., 102: 541-4, 1987.

3. BARROS, M.B.A. A utilização do conceito de classe social nos estudos dos perfis epidemiológicos: uma proposta. Rev. Saúde públ., S. Paulo, 20: 269-73, 1986.

4. BECERRA, J.E. \& SMITH, J.C. Matemal smoking and low birthweight in the reproductive history of women in Puerto Rico, 1982. Amer. J. publ., Hlth, 78: 268-72, 1988.

5. BELIZAN, J.M.; LECHTIG, A.; VILLAR, J. Distribution of low birth weight babies in developing countries. Amer. J. Obstet. Gynec., 132: 704-5, 1978.

6. BENICIO, M.H.D, A.; MONTEIRO, C.A.; SOUZA, J.M.P.; CASTILHO, E.A.; LAMONICA, I.M.R.; Análise multivariada de fatores de risco para o baixo peso ao nascer em nascidos vivos do município de São Paulo, SP (Brasil). Rev. Saúde públ., S. Paulo, 19: 311-20, 1985.

7. BETTIOL, H. Saúde perinatal em Ribeirão Preto: estudo de algumas variáveis sociais e biológicas no perfil reprodutivo de mães adolescentes. Ribeirão Preto, 1990. [Dissertação de Mestrado - Faculdade de Medicina de Ribeirão Preto da USP].

8. BEWLEY, B.R. Smoking in pregnancy. Brit. med J., 288: 424-6, 1984.

9. BUTLER, N.R \& ALBERMAN, E.D., eds. Perinatal problems. Edinburgh, E. \& S. Livinstone, 1969.

10. EVERITT, B.S. The analysis of contigency tables. New York, John Wiley \& Sons, 1977.

11. HEBEL, J.R; FOX, N.L.; SEXTON, M. Dose-response of birth weight to various measures of maternal smoking during pregnancy. J. clin. Epidem., 41: 483-9, 1988.

12. KRAMER, M.S. Determinants of low birth weight: methodological assessment and meta-analysis. Bull. Whd Hlth Org., 65: 663-737, 1987.

13. LEE, K.S; FERGUSON, R.M.; CORPUZ, M.; GARTNER, L.M. Matemal age and incidence of low birth weight at term: a population study. Amer. J. Obstet. Gynec., 158: 84-9, 1988.
14. MACARTHUR, C. \& ENOX, E.G. Smoking in pregnancy: effects of stopping at different stages. Brit. J. Obstet. Gynaec., 95: 551-5, 1988.

15. MONTEIRO, C.A.; ISHI, M.; BENICIO, M.H.D'A.; REA, M.F. A distribuição do peso ao nascer no município de São Paulo. Rev. Saúde públ., S. Paulo, 14: $161-72,1980$.

16. PICKERING, R.M. Relative risks of low birth weight in Scotland 1980-2. J. Epidem. Community Hlth, 41: 133-9, 1987.

17. ORGANIZACION PANAMERICANA DE LA SALUD. Fecundidad en la adolescencia: causas, riesgos y opciones. Washington, DC, 1988. (Cuademo Tecnico, 12).

18. ORGANIZACION PANAMERICANA DE LA SALUD. Salud del adolescente, prioridades y estrategias nacionales y regionales. Bol. Ofic. sanit. panamer., 107: 78-82, 1989.

19. ORUAMABO, R.S. \& JOHN, C.T. Antenatal care and fetal outcome, specially low birth-weight, in Port Harcourt, Nigeria. Ann trop. Paediatr., 3: 173-7, 1989.

20. PUFFER, R.R. \& SERRANO, C.V. Patterns of birth weights. Washington, DC, Pan American Health Organization, 1987. (PAHO-Scientific Publication, 504).

21. RUSH, D. \& CASSANO, P. Relationship of cigarette smoking and social class to birth weight and perinatal mortality among all births in Britain, 5-11 April 1970. J. Epidem. Community Hlth 37: 249-55, 1983.

22. SINGER, P.I. Dominação e desigualdade: estrutura de classes e repartição da renda no Brasil. Rio de Janeiro, Paz e Terra, 1981.

23. SPSS/PC + V 3.0: update manual. Chicago, SPSS Inc., 1988.

24. VICTORA, C.G.; BARROS, F.C.; MARTINES, J.C.; BORIA, J.U.; VAUGHAN, J.P. Estudo longitudinal das crianças nascidas em 1982, em Pelotas, RS, Brasil: metodologia e resultados preliminares. Rev. Saúde públ., S. Paulo, 19: 56-68, 1985.

25. WORLD HEALTH ORGANIZATION. The incidence of low birth weight: a critical review of available information. Wh Hlth Statist. Quart, 33: 197-224, 1980.
Recebido para publicaçāo em 3/9/1991 Reapresentado em 12/2/1992 Aprovado para publicaçāo em 1\&/2/1992 